WORKSHOP

Direct and Large-Eddy Simulation 11 May $29^{\text {th }}-31^{\text {th }} 2017$, Pisa, Italy

\title{
DIRECT NUMERICAL SIMULATION OF CONVECTIVE CHANNEL FLOW OF FLUID MIXTURES
}

\author{
P. Bahavar ${ }^{1}$, C. Wagner ${ }^{1,2}$ \\ 1 Institute of Aerodynamics and Flow Technology, German Aerospace Center (DLR) \\ 2 Institute of Thermodynamics and Fluid Mechanics, Technische Universität Ilmenau \\ philipp.bahavar@dlr.de
}

\section{INTRODUCTION}

Flows of fluid mixtures pose challenges to simulations due to the heterogeneous transport properties throughout the flow domain. For single-phase mixtures, the Boussinesq approximation, as routinely applied to model thermal buoyancy effects, can be adapted to provide a framework in which the fluid mixture can be described in a perturbative sense by calculating the density changes throughout the domain in terms of the local mixing ratio [1]. Here, Direct Numerical Simulations (DNS) are used to investigate this approach in turbulent channel flow with temperature differences, concentration differences, and a combination thereof to compare and contrast the resulting changes in the flow observables. Particular focus is placed on the mixture of water vapor and dry air, which has a wide range of applicability in both technical and naturally occurring systems, and is of interest regarding future investigation considering phase change phenomena in addition to the buoyant forces induced by the fluid heterogeneity.

\section{NUMERICAL METHODS}

The flow is described by the Navier-Stokes equations in the Boussinesq approximation for incompressible fluids,

$$
\begin{aligned}
\frac{\partial u_{i}}{\partial x_{i}} & =0 \\
\frac{\partial u_{i}}{\partial t}+\frac{\partial}{\partial x_{i}}\left(u_{i} u_{j}\right) & =-\frac{1}{\rho} \frac{\partial p}{\partial x_{i}}+\nu \frac{\partial^{2} u_{i}}{\partial x_{i} \partial x_{j}}-f_{i, \xi} \\
\frac{\partial \xi}{\partial t}+\frac{\partial}{\partial x_{i}}\left(u_{i} \xi\right) & =\phi_{\xi} \frac{\partial^{2} \xi}{\partial x_{i} \partial x_{j}}
\end{aligned}
$$

with density $\rho$, kinematic viscosity $\nu$, and buoyancy term $f_{i}$ depending on the scalar fields $\xi$ representing temperature $T$ or concentration $c$ with the corresponding diffusivities $\phi_{\xi}=\kappa, D$, respectively. In the Boussinesq approximation, the buoyant force term is given by

$$
\begin{gathered}
f_{i, \xi}=-\alpha_{\xi}\left(\xi-\xi_{\text {ref }}\right) g_{i}, \\
\alpha_{\xi}=\left.\frac{1}{\rho_{\text {ref }}} \frac{\partial \rho}{\partial \xi}\right|_{\xi_{\text {ref }}}
\end{gathered}
$$

in a linear approximation of the density dependence on the scalar $\xi$ around a reference value. The validity range for this leading order expansion depends on the properties of the fluids in question and has to be evaluated to ensure valid results [2].

For the simulation of turbulent channel flow, the computational domain with $x \in[0,5 \pi \delta], y \in[-\delta, \delta], z \in[-\pi \delta, \pi \delta]$, where $\delta$ is the channel half width, was represented in a mesh with $394 \times 180 \times 316$ hexahedral cells. For flows with Reynolds number $\mathrm{Re}=2280$ with corresponding friction Reynolds number $\operatorname{Re}_{\tau}=150$, the resulting mesh resolution for a uniform cell distribution is $\Delta x^{+}=6$ in streamwise and $\Delta z^{+}=3$ in spanwise direction, expressed in terms of wall units. Estimating the dissipation rate $\epsilon \simeq u_{b}^{3} / \delta$ with the bulk velocity $u_{b}$ yields

$$
\eta=\left(\frac{\nu^{3} \delta}{u_{b}}\right)^{\frac{1}{4}} \Rightarrow \eta^{+}=\left(\frac{u_{\tau}^{4} \delta}{u_{b}^{3} \nu}\right)^{\frac{1}{4}}=\frac{\operatorname{Re}_{\tau}}{\operatorname{Re}^{\frac{3}{4}}}=0.45
$$

for the Kolmogorov microscale. To resolve the large gradients in close proximity to the walls while minimizing computational effort, a hyperbolic tangens distribution is employed for the cell spacing in the wall-normal direction, resulting in $\Delta y_{\min }^{+}=$ 0.2 directly at the walls and $\Delta y_{\max }^{+}=3.7$ in the bulk flow. The presence of the scalar fields $\xi$ gives rise to the Batchelor scale, which is related to the Kolmogorov scale by $\lambda_{B}=\eta \sqrt{\phi_{\xi} / \nu}$. By choosing the Prandtl number $\operatorname{Pr}=\nu / \kappa<1$ and Schmidt number $\mathrm{Sc}=\nu / D<1, \lambda_{B}>\eta$ and the diffusive scales are automatically resolved as well.

In streamwise and spanwise direction cyclic boundaries are applied, while no-slip and impermeability boundary conditions are imposed on the velocity field at the channel walls. For the pressure equation, von-Neumann conditions are used, while the scalar fields are bounded by Dirichlet boundaries.

The flow equations are solved using a finite volume approach implemented in OpenFOAM [3]. To achieve the highest possible accuracy, fourth order discretization is employed for all spatial derivatives and interpolations, and an explicit second order Euler-Leapfrog integration scheme is used to advance the momentum equation in time. This imposes a strict upper bound for the time step $\Delta t$ to guarantee numerical stability [4]. The pressure is obtained by coupling pressure and velocity fields via the projection method, which ensures that the continuity equation is fulfilled. The resulting Poisson equation for the pressure is solved iteratively using a conjugate gradient method.

The accuracy of this implementation was verified by comparing results for the first and second statistical moments of both velocity and temperature with results from spectral DNS [5]. Figure 1 shows exemplary the comparison for the mean streamwise velocity and fluctuations, averaged over the homogeneous spatial directions. Agreement is exact for the velocity profiles and the fluctuations close to the walls, with visible underestimation of the fluctuations in the bulk region. 

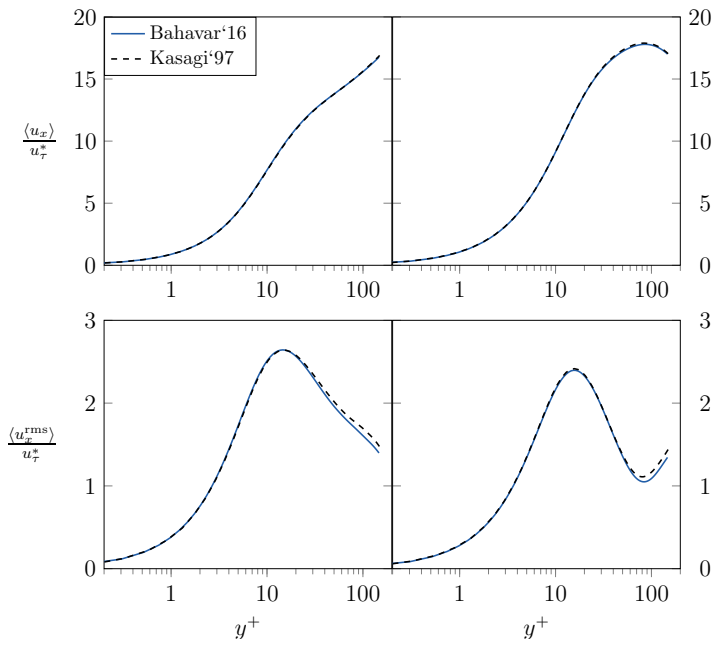

Figure 1: Results for the mean streamwise velocity (top) and fluctuations (bottom) compared to profiles from spectral DNS [5] for a differentially heated channel at the cold wall (left panels) and the hot wall (right).

\section{FLUID MIXTURE RESULTS}

Simulations for a mixture of two fluids were performed using the approach outlined above. The scalar transport is characterized by $\mathrm{Pr}=0.71$ and $\mathrm{Sc}=0.48$ corresponding to water vapor in a carrier fluid of dry air. The limits of the Boussinesq approximation are calculated to be $\Delta T<27 \mathrm{~K}$ and $\Delta c=0.15$ for the temperature and concentration field, respectively, and Grashof numbers

$$
\mathrm{Gr}=\frac{g \delta^{3}}{\nu^{2}} \sum_{\xi=T, c} \phi_{\xi} \Delta \xi
$$

are chosen according to values within the range of applicability, $\mathrm{Gr}=(1.20,1.51,2.44) \cdot 10^{5}$, by setting fixed value boundary conditions for the scalar fields at the opposing channel walls. The thermal gradient is constant across all simulated cases, such that the increase in the Grashof number is caused by an increased concentration difference only. The density gradients caused by both scalar fields align, causing the buoyant forces to add linearly. Figure 2 shows the results of the three different systems in comparison to turbulent channel flow without additional buoyant forces. The acceleration/deceleration of the mean flow is clearly visible in the velocity profiles, showing that for a mixture of water vapor and dry air, the effects caused by the concentration difference are of the same order of magnitude as those with thermal origin for differences within the validity range of the Boussinesq approximation. Fluctuations in the streamwise velocity are increased over a channel cross section growing with $\mathrm{Gr}$ and extending far across the center plane. A decrease is visible only in close proximity to the wall where the buoyancy aids the flow, where two local minima exist for the lower values of $\mathrm{Gr}$, changing into one minimum as the increase of the bulk fluctuations moves towards the aiding wall at the highest value.
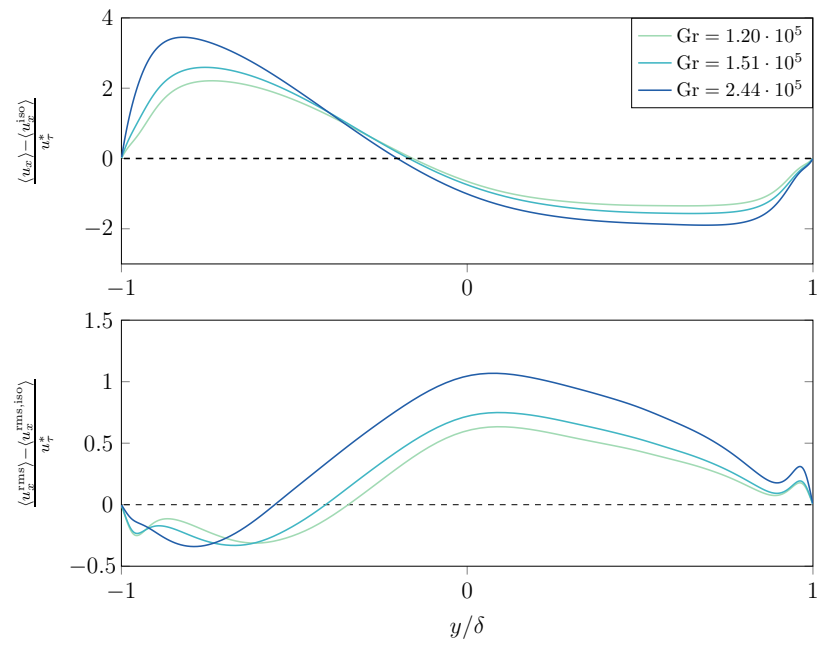

Figure 2: Differences in mean streamwise velocity (top) and fluctuations (bottom) of buoyant flow compared to isothermal and single-component flow, for increasing values of $\mathrm{Gr}$.

\section{CONCLUSIONS}

A finite volume DNS was used to simulate turbulent channel flow of a differentially heated channel containing a mixture of two fluids. To this end, a perturbative approach to the simulation of the fluid mixture with different densities was demonstrated and compared to the well-established treatment of thermal differentials within a single fluid species. The results for a mixture of water vapor and dry air show that the additional buoyancy stemming from concentration differences leads to non-negligible effects in the flow observables. The influence an increasing concentration difference follows the same behavior expected from larger temperature differences, as is expected from the analogous formulation of both the transport equation as well as the buoyancy contribution to the momentum equation.

Using the highly resolved temperature and concentration information available throughout the channel, it is possible to identify regions of oversaturation where a phase transition may occur. An investigation of the influence of the added heat flow from condensation is then possible without the added computational cost of a full multiphase simulation.

\section{REFERENCES}

[1]Gebhart, B. and Pera, L. : The nature of vertical natural convection flows resulting from the combined buoyancy effects of thermal and mass diffusion, Int. J. Heat Mass Transfer, 14, 12, 20252050 (1971).

[2]Gray, D.D. and Giorgini, A. : The validity of the Boussinesq approximation for liquids and gases, Int. J. Heat Mass Transfer, 19, 5, 545-511 (1976).

[3]Kath, C. and Wagner, C. : Highly Resolved Simulations of Turbulent Mixed Convection in a Vertical Plane Channel, New Results in Numerical and Experimental Fluid Mechanics, 10, 515-524 (2016).

[4]Shishkina, O. and Wagner, C. : Stability conditions for the leapfrog-euler scheme with central spatial discretization of any order, Appl. Num. Anal. Comp. Math., 1, 1, 315-326 (2004).

[5]Kasagi, N. and Nishimura, M. : Direct numerical simulation of combined forced and natural turbulent convection in a vertical plane channel, Int. J. Heat Fluid Flow, 18, 1, 88-99 (1997). 